\title{
Ranging in a Single-Input Multiple-Output (SIMO) System
}

\author{
Sinan Gezici, Member, IEEE, and Zafer Sahinoglu, Senior Member, IEEE
}

\begin{abstract}
In this letter, optimal ranging in a single-input multiple-output (SIMO) system is studied. The theoretical limits on the accuracy of time-of-arrival (TOA) (equivalently, range) estimation are calculated in terms of the Cramer-Rao lower bound (CRLB). Unlike the conventional phased array antenna structure, a more generic fading model is employed, which allows for the analysis of spatial diversity gains from the viewpoint of a ranging system. In addition to the optimal solution, a two-step suboptimal range estimator is proposed, and its performance is compared with the CRLBs.
\end{abstract}

Index Terms-Time-of-arrival (TOA) estimation, Cramer-Rao lower bound (CRLB), single-input multiple-output (SIMO) systems.

\section{INTRODUCTION}

$\mathbf{U}$ SE of multiple-input multiple-output (MIMO) architectures is becoming a common approach for high speed wireless systems. By means of multiple antennas and multiple processing units for different antennas, quality of communications between wireless devices can be increased via diversity and multiplexing techniques. Although the advantages of such MIMO structures have been studied extensively for communications systems [1], they have not been investigated in detail from the viewpoint of positioning systems. Commonly, multiple antenna elements are closely spaced together to form phased array structures in radar and positioning applications [2]. Recently, the advantages of the MIMO approach for radar systems were studied in [3]. Since then, MIMO systems have been considered for radar applications for better detection and characterization of target objects.

The aim of this paper is to quantify the advantages of MIMO structures for positioning applications, and to introduce the concept of diversity for range (TOA) estimation. Specifically, a SIMO system is considered as a first step, and the benefits of diversity for ranging is quantified by means of CRLBs. In addition, a practical range estimator with low computational complexity is proposed, and its performance is investigated via theoretical and numerical calculations. It is shown that the proposed estimator approximately achieves the CRLB at high signal-to-noise ratios (SNRs).

\section{Signal MOdel AND CRLBS}

Consider a SIMO system with $N$ receive antenna elements, and assume that the maximum distance between the antenna pairs divided by the speed of light is considerably smaller than the symbol duration. Then, the baseband received signal at the $i$ th antenna can be expressed as

$$
r_{i}(t)=\alpha_{i} s(t-\tau)+n_{i}(t), \quad t \in[0, T],
$$

Manuscript received October 15, 2007. The associate editor coordinating the review of this letter and approving it for publication was F. Jondral. This research was supported in part by the EU FP7 Projects WiMAGIC under grant no. 215167 and NEWCOM++ under grant no. 216715 .

S. Gezici is with the Department of Electrical and Electronics Engineering, Bilkent University, Bilkent, Ankara TR-06800, Turkey (e-mail: gezici@ee.bilkent.edu.tr).

Z. Sahinoglu is with Mitsubishi Electric Research Labs, 201 Broadway, Cambridge, MA 02139, USA (e-mail: zafer@merl.com).

Digital Object Identifier 10.1109/LCOMM.2008.071691. for $i=1, \ldots, N$, where $s(t)$ is the baseband representation of the transmitted signal, $\alpha_{i}$ is the channel coefficient of the received signal at the $i$ th antenna, $\tau$ is the TOA, and $n_{i}(t)$ is a complex-valued white Gaussian noise process with zero mean and spectral density $\sigma_{i}^{2}$. It is assumed that noise processes at different receiver branches are independent, and that there is sufficient separation (comparable to the signal wavelength) between all antenna pairs so that different channel coefficients can be observed at different antennas. This is unlike a phased array structure in which $\alpha_{i}=\alpha \forall i$.

The ranging problem in a SIMO system involves the estimation of the TOA $\tau$ from the received signals at $N$ receive antennas. In addition, the channel coefficients $\boldsymbol{\alpha}=\left[\alpha_{1} \cdots \alpha_{N}\right]$ are also unknown, and need to be considered in the estimation problem in general. If the complex channel coefficients are represented as $\alpha_{i}=a_{i} e^{j \phi_{i}}$ for $i=1, \ldots, N$, the vector of unknown signal parameters can be expressed as $\boldsymbol{\lambda}=\left[\begin{array}{lll}\tau & \boldsymbol{a} & \boldsymbol{\phi}\end{array}\right]$, where $\boldsymbol{a}=\left[a_{1} \cdots a_{N}\right]$ and $\boldsymbol{\phi}=\left[\phi_{1} \cdots \phi_{N}\right]$.

From (1), the log-likelihood function for $\boldsymbol{\lambda}$ can be expressed as [4]

$$
\Lambda(\boldsymbol{\lambda})=k-\sum_{i=1}^{N} \frac{1}{2 \sigma_{i}^{2}} \int_{0}^{T}\left|r_{i}(t)-\alpha_{i} s(t-\tau)\right|^{2} \mathrm{~d} t
$$

where $k$ represents a term that is independent of $\lambda$. Then, the maximum likelihood (ML) estimate for $\boldsymbol{\lambda}$ can be obtained from (2) as

$\hat{\boldsymbol{\lambda}}_{\mathrm{ML}}=\arg \max _{\boldsymbol{\lambda}} \sum_{i=1}^{N} \frac{1}{\sigma_{i}^{2}} \int_{0}^{T} \mathcal{R}\left\{\alpha_{i}^{*} r_{i}(t) s^{*}(t-\tau)\right\} \mathrm{d} t-\frac{E\left|\alpha_{i}\right|^{2}}{2 \sigma_{i}^{2}}$

where $E=\int_{-\infty}^{\infty}|s(t)|^{2} \mathrm{~d} t$ is the signal energy ${ }^{1}$.

From (2), the Fisher information matrix (FIM) [4] can be obtained, after some manipulation, as

$$
\mathbf{I}=\left[\begin{array}{lll}
\mathbf{I}_{\tau \tau} & \mathbf{I}_{\tau a} & \mathbf{I}_{\tau \phi} \\
\mathbf{I}_{\tau a}^{T} & \mathbf{I}_{a a} & \mathbf{I}_{a \phi} \\
\mathbf{I}_{\tau \phi}^{T} & \mathbf{I}_{a \phi}^{T} & \mathbf{I}_{\phi \phi}
\end{array}\right]
$$

with

$$
\begin{aligned}
\mathrm{I}_{\tau \tau} & =\tilde{E} \sum_{i=1}^{N} \frac{\left|\alpha_{i}\right|^{2}}{\sigma_{i}^{2}}, \\
\mathbf{I}_{a a} & =\operatorname{diag}\left\{E / \sigma_{1}^{2}, \ldots, E / \sigma_{N}^{2}\right\}, \\
\mathbf{I}_{\phi \phi} & =\operatorname{diag}\left\{E\left|\alpha_{1}\right|^{2} / \sigma_{1}^{2}, \ldots, E\left|\alpha_{N}\right|^{2} / \sigma_{N}^{2}\right\}, \\
\mathbf{I}_{\tau a} & =-\left[\hat{E}_{\mathrm{R}}\left|\alpha_{1}\right| / \sigma_{1}^{2} \cdots \hat{E}_{\mathrm{R}}\left|\alpha_{N}\right| / \sigma_{N}^{2}\right], \\
\mathbf{I}_{\tau \phi} & =-\left[\hat{E}_{\mathrm{I}}\left|\alpha_{1}\right|^{2} / \sigma_{1}^{2} \cdots \hat{E}_{\mathrm{I}}\left|\alpha_{N}\right|^{2} / \sigma_{N}^{2}\right], \\
\mathbf{I}_{a \phi} & =\mathbf{0}
\end{aligned}
$$

where $\operatorname{diag}\left\{x_{1}, \ldots, x_{N}\right\}$ represents an $N \times N$ diagonal matrix with its $i$ th diagonal being equal to $x_{i}, \tilde{E}$ is the energy of the first derivative of $s(t)$; i.e., $\tilde{E}=\int_{-\infty}^{\infty}\left|s^{\prime}(t)\right|^{2} \mathrm{~d} t$, and $\hat{E}_{\mathrm{R}}$ and

\footnotetext{
${ }^{1}$ For a complex number $z, \mathcal{R}\{z\}$ and $\mathcal{I}\{z\}$ represent its real and imaginary parts, respectively.
} 
$\hat{E}_{\mathrm{I}}$ are given, respectively, by

$$
\hat{E}_{\mathrm{R}}=\int_{-\infty}^{\infty} \mathcal{R}\left\{s^{\prime}(t) s^{*}(t)\right\} \mathrm{d} t, \hat{E}_{\mathrm{I}}=\int_{-\infty}^{\infty} \mathcal{I}\left\{s^{\prime}(t) s^{*}(t)\right\} \mathrm{d} t .
$$

From the formula for block matrix inversion, the first element of the inverse of $\mathbf{I},\left[\mathbf{I}^{-1}\right]_{11}$, can be obtained after some manipulation ${ }^{2}$. Then, the CRLB for unbiased delay estimates can be expressed as

$$
\operatorname{Var}\{\hat{\tau}\} \geq\left[\mathbf{I}^{-1}\right]_{11}=\frac{1}{\gamma \sum_{i=1}^{N} \frac{\left|\alpha_{i}\right|^{2}}{\sigma_{i}^{2}}},
$$

where $\gamma \doteq \tilde{E}-\hat{E}^{2} / E$, with

$$
\hat{E}=\left|\int_{-\infty}^{\infty} s^{\prime}(t) s^{*}(t) \mathrm{d} t\right| .
$$

In the case of known channel coefficients, it can be shown from (5) that the CRLB for delay estimation is as in (12) except that $\gamma$ is replaced by $\tilde{E}$. This simple observation implies that for signals with $\gamma=\tilde{E}$ (i.e., $\hat{E}=0$ ), the TOA estimation accuracy limit is the same for both known and unknown channel cases. In other words, the same estimation accuracy can be obtained even in the absence of channel state information for certain types of signals. For example, if $s(t)$ is a real and even function of time, $\hat{E}$ can be shown to be equal to zero, and $\gamma$ in (12) can be replaced by $\tilde{E}$.

In order to compare the previous analysis with a conventional phased array structure, consider closely-spaced antenna elements that result in the following signal model:

$$
r_{i}(t)=\alpha s(t-\tau)+n_{i}(t), \quad t \in[0, T],
$$

for $i=1, \ldots, N$. The only difference of (14) from (1) is the constant channel coefficient for all the signals received at the antennas. In this case, the vector of unknown parameters reduces to $\boldsymbol{\lambda}=\left[\begin{array}{ll}\tau & a\end{array}\right]$, where $\alpha=a e^{j \phi}$. By similar calculations that lead to (4), the FIM for the phased array case can be obtained as

$$
\mathbf{I}=\sum_{i=1}^{N} \frac{1}{\sigma_{i}^{2}}\left[\begin{array}{ccc}
\tilde{E}|\alpha|^{2} & -\hat{E}_{\mathrm{R}}|\alpha| & -\hat{E}_{\mathrm{I}}|\alpha|^{2} \\
-\hat{E}_{\mathrm{R}}|\alpha| & E & 0 \\
-\hat{E}_{\mathrm{I}}|\alpha|^{2} & 0 & E|\alpha|^{2}
\end{array}\right] .
$$

Then, the CRLB can be expressed as

$$
\operatorname{Var}\{\hat{\tau}\} \geq \frac{1}{\gamma|\alpha|^{2} \sum_{i=1}^{N} \frac{1}{\sigma_{i}^{2}}} .
$$

In the case of known channel coefficient $\alpha, \gamma$ in (16) is replaced by $\tilde{E}$.

Comparison of (12) and (16) reveals that the CRLB is more robust to channel fading for the SIMO system, since the channel dependent term in the denominator of (12) is more robust to channel variations. In the case of a phased array, a significantly fading signal path can result in a quite large CRLB as can be observed from (16). In other words, similar to the diversity gain for communications systems, multiple receive antennas can also provide diversity for ranging systems.

For the case of known channel coefficients and $\sigma_{i}=\sigma \forall i$, (12) and (16) can be expressed in terms of the effective bandwidth $\beta, \beta^{2} \doteq \frac{1}{E} \int_{-\infty}^{\infty} f^{2}|S(f)|^{2} \mathrm{~d} f$, with $S(f)$ representing the Fourier transform of $s(t)$, as

\footnotetext{
${ }^{2}$ For $\mathbf{I}=\left[\begin{array}{cc}\mathbf{A} & \mathbf{B} \\ \mathbf{B}^{T} & \mathbf{D}\end{array}\right],\left[\mathbf{I}^{-1}\right]_{M \times M}=\left(\mathbf{A}-\mathbf{B D}^{-1} \mathbf{B}^{T}\right)^{-1}$, where $\mathbf{A}$ is an $M$-by- $M$ matrix
}

$$
\begin{aligned}
& \sqrt{\operatorname{Var}\{\hat{d}\}} \geq \frac{c}{2 \pi \beta \sqrt{\sum_{i=1}^{N} \mathrm{SNR}_{i}}}, \\
& \sqrt{\operatorname{Var}\{\hat{d}\}} \geq \frac{c}{2 \pi \sqrt{N} \beta \sqrt{\mathrm{SNR}}},
\end{aligned}
$$

respectively, where $\hat{d}$ is an unbiased range estimate obtained from delay estimation, $c$ is the speed of light, and the signal-to-noise ratios are defined as $\operatorname{SNR}_{i}=\left|\alpha_{i}\right|^{2} E / \sigma^{2}$ for $i=1, \ldots, N$, and SNR $=|\alpha|^{2} E / \sigma^{2}$. Note that (18) is the conventional CRLB expression for ranging systems [5] scaled by $1 / \sqrt{N}$ due to the presence of multiple receive antennas. Again the diversity provided by the SIMO structure can be observed from (17).

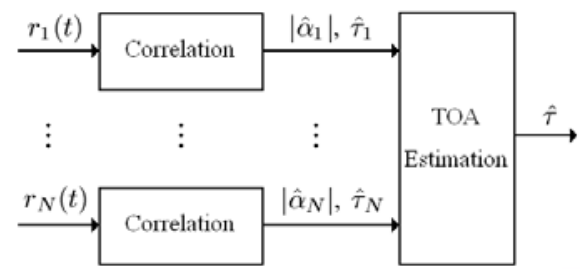

Fig. 1. An asymptotically optimal algorithm for joint TOA and range estimation.

\section{A Practical Ranging Algorithm}

\section{A. Algorithm Description}

In general, the ML solution in (3) requires optimization over an $(N+1)$-dimensional space, which can have prohibitive complexity in scenarios with a large number of receive antennas. In this section, a two-step suboptimal estimator, as shown in Figure 1, is proposed, which performs joint channel and delay estimation at each output branch in the first step, and implements a simple delay (range) estimator in the second step. Note that the algorithm exploits the multiple-output structure of a SIMO system, which facilitates individual signal processing, such as correlation or matched filter based channel coefficient and delay estimation, at each receiver branch.

In the first step of the estimator, each branch processes its received signal individually, and provides estimates of the channel coefficient and the delay, based on an ML approach. For the $i$ th branch, the ML estimates of $\alpha_{i}\left(=a_{i} e^{j \phi_{i}}\right)$ and $\tau$ can be obtained from $r_{i}(t)$ in (1) as follows:

$$
\begin{aligned}
& \left(\hat{\tau}_{i}, \hat{\phi}_{i}\right)=\arg \max _{\tau, \phi_{i}} \mathcal{R}\left\{e^{-j \phi_{i}} \int_{0}^{T} r_{i}(t) s^{*}(t-\tau) \mathrm{d} t\right\}, \\
& \hat{a}_{i}=\left|\hat{\alpha}_{i}\right|=\frac{1}{E} \mathcal{R}\left\{e^{-j \hat{\phi}_{i}} \int_{0}^{T} r_{i}(t) s^{*}\left(t-\hat{\tau}_{i}\right) \mathrm{d} t\right\},
\end{aligned}
$$

for $i=1, \ldots, N$. Note that the ML estimation results in a correlator, as in (19), which provides the delay and phase estimates; and the channel amplitude can be directly estimated from those estimates as in (20).

In the second step, the estimates for the channel amplitudes and the delays are used to estimate the TOA as follows:

$$
\hat{\tau}=\frac{\sum_{i=1}^{N} \widehat{\operatorname{SNR}}_{i} \hat{\tau}_{i}}{\sum_{i=1}^{N} \widehat{\operatorname{SNR}}_{i}},
$$

where $\widehat{\operatorname{SNR}}_{i}=E\left|\hat{\alpha}_{i}\right|^{2} / \sigma_{i}^{2}$. In other words, the TOA is estimated as a weighted average of the delay estimates obtained at the $N$ receiver branches, where the weights are proportional to the SNR estimates at the respective branches. 


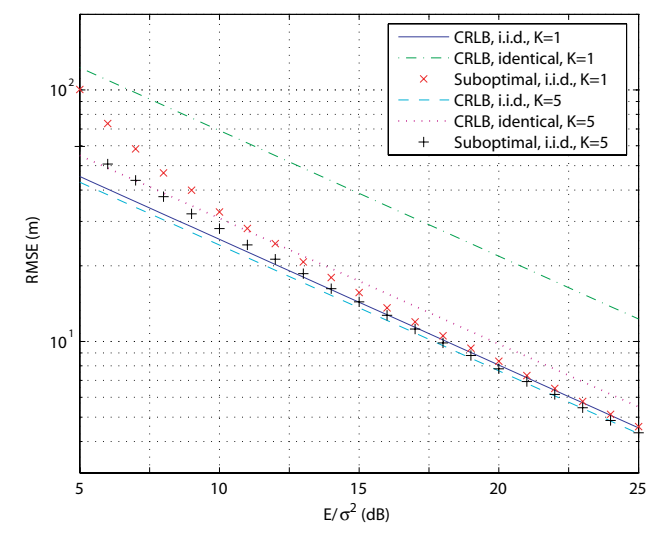

Fig. 2. The RMSE of the two-step algorithm and the CRLBs.

\section{B. Complexity and Performance}

The computational complexity of the two-step estimator in Figure 1 is dominated by the optimization operations in (19). In other words, the estimator requires the solution of $N$ optimization problems, each over a 2-dimensional space. On the other hand, the optimal ML solution in (3) requires optimization over an $(N+1)$-dimensional space, which is computationally more complex than the proposed algorithm. In fact, as $N$ increases, the optimal solution becomes quite impractical.

The reduction in the computational complexity of the twostep algorithm results in its suboptimality in general compared to the ML algorithm in (3). However, under certain circumstances, it can be shown that the two-step scheme performs very closely to the optimal solution; i.e., it approximately achieves the CRLB of the original problem.

To this end, first consider the following lemma, which provides an approximate model for the estimates in (19) and (20) under certain conditions.

Lemma 1: For the signal model in (1) with $\hat{E}=0$ (cf. (13)), the delay estimate in (19) and the channel amplitude estimate in (20) can be modeled, at high SNR, as

$$
\begin{aligned}
\hat{\tau}_{i} & =\tau+\nu_{i}, \\
\left|\hat{\alpha}_{i}\right| & =\left|\alpha_{i}\right|+\eta_{i},
\end{aligned}
$$

for $i=1, \ldots, N$, where $\nu_{i}$ and $\eta_{i}$ are independent zero mean Gaussian random variables with variances $\sigma_{i}^{2} /\left(\tilde{E}\left|\alpha_{i}\right|^{2}\right)$ and $\sigma_{i}^{2} / E$, respectively. In addition, $\nu_{i}$ and $\nu_{j}\left(\eta_{i}\right.$ and $\left.\eta_{j}\right)$ are independent for $i \neq j$.

Proof: From the signal model in (1), the log-likelihood function can be expressed as

$$
\Lambda(\boldsymbol{\theta})=k_{i}-\frac{1}{2 \sigma_{i}^{2}} \int_{0}^{T}\left|r_{i}(t)-\alpha_{i} s(t-\tau)\right|^{2} \mathrm{~d} t,
$$

where $\boldsymbol{\theta}=\left[\begin{array}{lll}\tau & a_{i} \phi_{i}\end{array}\right]$, with $\alpha_{i}=a_{i} e^{j \phi_{i}}$.

Similar to the proof in [6] for obtaining the statistics of multipath delay estimates, one can approximate the loglikelihood function evaluated at the ML estimate $\hat{\boldsymbol{\theta}}, \Lambda(\hat{\boldsymbol{\theta}})$, by two terms from its Taylor series expansion around $\boldsymbol{\theta}$ for high SNRs. Then the ML estimate $\hat{\boldsymbol{\theta}}$ can be approximated by a multivariate Gaussian random variable with mean $\boldsymbol{\theta}$ and the covariance matrix given by the inverse of $\frac{\partial \Lambda(\boldsymbol{\theta})}{\partial \boldsymbol{\theta}}\left(\frac{\partial \Lambda(\boldsymbol{\theta})}{\partial \boldsymbol{\theta}}\right)^{T}$.

Therefore, the covariance matrix of the ML estimate can be obtained after some manipulation as $\operatorname{diag}\left\{\sigma_{i}^{2} /\left(\tilde{E}\left|\alpha_{i}\right|^{2}\right), \sigma_{i}^{2} / E, \sigma_{i}^{2} / E\left|\alpha_{i}\right|^{2}\right\}$, for $\hat{E}=0$.

Since the estimates in (19) and (20) are the ML estimates according to the signal model in (1), the result of the lemma follows. Also, since the noise processes are independent at different receiver branches, the noise components for different branches are independent as stated in the lemma.

Lemma 1 establishes the approximate unbiasedness and efficiency of the two-step estimator, as implied by the following proposition.

Proposition 1: For the delay and channel amplitude estimates as modeled in Lemma 1, the TOA estimator in (21) is an unbiased estimator of $\tau$ with the following variance

$$
\operatorname{Var}\{\hat{\tau}\}=\frac{1}{\tilde{E}} \mathrm{E}\left\{\sum_{i=1}^{N} \frac{\left|\hat{\alpha}_{i}\right|^{4}}{\sigma_{i}^{2}\left|\alpha_{i}\right|^{2}}\left(\sum_{i=1}^{N} \frac{\left|\hat{\alpha}_{i}\right|^{2}}{\sigma_{i}^{2}}\right)^{-2}\right\},
$$

where the expectation is over $\left|\hat{\alpha}_{i}\right|$ 's modeled by (23).

Proof: Conditioned on the channel estimates, the expected value of $\hat{\tau}$ in (21) can be shown to be equal to $\tau$ under the model in (22), which proves the unbiasedness property. Similarly, the variance can be obtained as in the proposition ${ }^{3} . \square$

Note that the variance of the two-step estimator in (25) is always larger than the CRLB in (12). However, as $E / \sigma_{i}^{2}$ gets higher, $\left|\hat{\alpha}_{i}\right|$ gets closer to $\left|\alpha_{i}\right|$ (Lemma 1), and the variance in (25) becomes approximately equal to the CRLB for $\hat{E}=0$.

\section{RESULTS}

In order to compare CRLBs for generic SIMO systems and phased arrays, and to analyze performance of the proposed two-step algorithm in Section III, a uniform linear array (ULA) structure with $N=5$ antennas is considered for a narrowband signal with $1 \mathrm{MHz}$ bandwidth and $3 \mathrm{GHz}$ carrier frequency. The channel is modeled to be Rician fading with a $K$-factor of $K$, and it is assumed that the average noise power is the same at all the receiver branches; i.e., $\sigma_{i}=\sigma \forall i$.

In Figure 2, the RMSEs of the two-step algorithm ("suboptimal") are plotted for $K=1$ and $K=5$, together with the CRLBs for the case of i.i.d. fading channel coefficients at different receiver branches ${ }^{4}$. Also shown in the figure are the CRLBs for the phased array case, in which the antenna elements are closely spaced together so that the channel coefficients are identical at all the antennas.

It is observed from the figure that the accuracy is better in the i.i.d. fading scenarios than that in the phase array scenarios, especially for the cases without strong line-of-sight components (i.e., for small $K \mathrm{~s}$ ). In addition, the two-step algorithm converges to the CRLB at high SNRs as expected, although it does not perform that well at low SNRs.

\section{REFERENCES}

[1] D. Tse and P. Viswanath, Fundamentals of Wireless Communications. Cambridge University Press, 2005.

[2] M. A. Richards, Fundamentals of Radar Signal Processing, 1st ed. New York: McGraw-Hill, 2005.

[3] E. Fishler, A. Haimovich, R. Blum, L. Cimini, D. Chizhik, and R. Valenzuela, "MIMO radar: an idea whose time has come," in Proc. IEEE Int. Conf. on Radar, Philadelphia, PA, Apr. 2004.

[4] H. V. Poor, An Introduction to Signal Detection and Estimation, 2nd ed. New York: Springer-Verlag, 1994.

[5] S. Gezici, Z. Tian, G. B. Giannakis, H. Kobayashi, A. F. Molisch, H. V. Poor, and Z. Sahinoglu, "Localization via ultra-wideband radios," IEEE Signal Processing Mag., vol. 22, no. 4, pp. 70-84, July 2005.

[6] Y. Qi, H. Kobayashi, and H. Suda, "On time-of-arrival positioning in a multipath environment," IEEE Trans. Veh. Technol., vol. 55, no. 5, pp. 1516-1526, Sept. 2006.

\footnotetext{
${ }^{3}$ The details are omitted due to the space limitation.

${ }^{4}$ The antennas are spaced $10 \mathrm{~cm}$ apart in the i.i.d. fading case.
} 